\title{
Is food addiction a predictor of treatment outcome among patients with eating disorder?
}

\author{
Xandra Romero $^{1,2}$ (i) | Zaida Agüera ${ }^{2,3,4}$ (i) | Roser Granero ${ }^{3,5}$ (i) | Isabel Sánchez ${ }^{2,3}$ (i) | \\ Nadine Riesco $^{2,3}$ (D) | Susana Jiménez-Murcia ${ }^{1,2,3}$ (D) | Montserrat Gisbert-Rodriguez ${ }^{2}$ | \\ Jéssica Sánchez-González ${ }^{1,2}$ | Gemma Casalé2 (D) | Isabel Baenas ${ }^{2}$ (D) | \\ Eduardo Valenciano-Mendoza ${ }^{2}$ | Jose M. Menchon ${ }^{1,2,6}$ (i) | Ashley N. Gearhardt ${ }^{7}$ (i) | \\ Carlos Dieguez $^{3,8}$ (D) | Fernando Fernández-Aranda ${ }^{1,2,3}$ (D)
}

${ }^{1}$ Department of Clinical Sciences, School of Medicine and Health Sciences, University of Barcelona, Barcelona, Spain
${ }^{2}$ Department of Psychiatry, University Hospital of Bellvitge-IDIBELL, Barcelona, Spain
${ }^{3}$ CIBER Fisiopatología Obesidad y Nutrición (CIBERobn), Instituto Salud Carlos III, Barcelona, Spain
${ }^{4}$ Department of Public Health, Mental Health and Maternal-Child Nursing, School of Nursing, University of Barcelona, Barcelona, Spain
${ }^{5}$ Departament de Psicobiologia i Metodologia de les Ciències de la Salut, Universitat Autònoma de Barcelona, Barcelona, Spain
${ }^{6}$ CIBER Salud Mental (CIBERSAM), Instituto Salud Carlos III, Barcelona, Spain
${ }^{7}$ Department of Psychology, University of Michigan, Ann Arbor, Michigan
${ }^{8}$ Department of Physiology, CIMUS, University of Santiago de Compostela-Instituto de Investigación Sanitaria, Santiago de Compostela, Spain

\section{Correspondence}

Fernando Fernández-Aranda, PhD,

FAED, Department of Psychiatry,

University Hospital of Bellvitge-

CIBEROBN, c/Feixa Llarga s/n,

L'Hospitalet de Llobregat, Barcelona

08907, Spain.

Email: f.fernandez@ub.edu

Zaida Agüera, PhD, CIBEROBN and Department of Psychiatry, University Hospital of Bellvitge, c/Feixa Llarga s/n,

L'Hospitalet de Llobregat, Barcelona 08907, Spain.

Email: zaguera@bellvitgehospital.cat

\section{Funding information}

European Regional Development Fund, Grant/Award Number: ERDF; Generalitat de Catalunya, Grant/Award Numbers: CERCA programme and SLT006/17/ 00077; Instituto de Salud Carlos III, Grant/Award Numbers: CIBERSAM, CIBERobn and PI17/01167; Ministerio de Economía y Competitividad, Grant/Award Number: PSI2015-68701-R; Ministerio de Sanidad, Servicios Sociales e Igualdad, Grant/Award Number: PR338/17; Delegación del Gobierno para el Plan

\begin{abstract}
Objectives: The study aimed to examine whether food addiction (FA) was associated with greater severity in both binge eating disorders (BED) and bulimia nervosa and, therefore, to determine if FA was predictive of treatment outcome.

Method: Seventy-one adult patients with bulimia nervosa and BED (42 and 29, respectively) participated in the study. FA was assessed by means of the Yale Food Addiction Scale.

Results: The results confirmed a high prevalence of FA in patients with binge disorders (around 87\%) and also its association with a greater severity of the disorder (i.e., related to an increased eating psychopathology and greater frequency of binge eating episodes). Although FA did not appear as a predictor of treatment outcome in general terms, when the diagnostic subtypes were considered separately, FA was associated with poor prognosis in the BED group. In this vein, FA appeared as a mediator in the relationship between ED severity and treatment outcome.
\end{abstract}

Discussions: Our findings suggest that FA may act as an indicator of ED severity, and it would be a predictor of treatment outcome in BED but not in BN. 
Nacional sobre Drogas, Grant/Award Number: 2017I067

\section{KEYWORDS}

binge eating disorder, bulimia nervosa, eating disorders, food addiction, treatment outcome

\section{1 | INTRODUCTION}

Food addiction (FA) is a controversial construct that has not been recognized as a diagnostic entity so far. However, FA is receiving an increased interest and a growing body of research in the last years (FernándezAranda, 2018). The concept of FA posits that individuals experience addictive-like symptoms related to the consumption of high-calorie/palatable foods (Bonder, Davis, Kuk, \& Loxton, 2018; Davis, 2016; Meule, von Rezori, \& Blechert, 2014; such as preoccupation with obtaining a desired food, excessive consumption of unhealthy foods despite adverse health consequences, craving, impaired control, tolerance, withdrawal, and distress/dysfunction; Fletcher \& Kenny, 2018; Gordon, Ariel-Donges, Bauman, \& Merlo, 2018), and with biological-hormonal maintaining factors (Novelle \& Diéguez, 2018; Peters et al., 2018). It has been related to other addictive disorders due to the parallels in the neurochemistry (Gearhardt 2016; Jiménez-Murcia et al., 2017; Tomasi et al., 2015), specifically in terms of participation of the dopaminergic reward system and the activation of positive reinforcement mechanisms (Carter \& Davis, 2011; Racine, Hagan, \& Schell, 2019; Volkow, Wang, Fowler, Tomasi, \& Baler, 2012). This evidence suggests that FA could be evaluated by considering the behavioural and symptomatology patterns observed in other addictions, as captured in the Yale Food Addiction Scale (YFAS; Gearhardt, Corbin, \& Brownell, 2009). Nevertheless, the debate about FA vs. eating addiction vs. nonaddiction remains open (Meule, 2019). Although some researchers have postulated for an integration of FA into substance use disorders (Gordon et al., 2018), others argue that it would be within behavioural addictions (Albayrak, Wölfle, \& Hebebrand, 2012). On the other hand, other studies even doubt about the existence of underlying addictive processes and postulate the loss of control overeating as a core symptom of the eating disorder (ED; Fletcher \& Kenny, 2018).

Despite FA has not yet been formally recognized by the Diagnostic and Statistical Manual for Mental Disorders (DSM-5; APA, 2013), it has emerged as a clinical entity recognized within the spectrum of obesity and abnormal eating behaviour (Wiss \& Brewerton, 2017). Recent studies have shown a high prevalence of FA among patients with obesity and/or with an ED, especially among those with binge eating symptomatology (Granero et al., 2014; Granero et al., 2018; Wiss \&
Brewerton, 2017). Thus, studies on ED have found a high prevalence of FA in patients with bulimia nervosa (BN; de Vries \& Meule, 2016; Granero et al., 2018; Meule et al., 2014) and binge eating disorder (BED; Gearhardt, Boswell, \& White, 2014; Penzenstadler et al. 2018; Smith and Robbins 2013), reaching rates ranging from $70 \%$ to $90 \%$ (Granero et al., 2014). In addition, FA has also been associated with higher eating-related severity and psychopathology (Granero et al., 2014; Imperatori et al., 2014), especially with greater levels of weight/shape overvaluation and body dissatisfaction, an increased frequency of binge eating episodes (Burrows, Hides, Brown, Dayas, \& Kay-Lambkin, 2017; Burrows, Kay-Lambkin, Pursey, Skinner, \& Dayas, 2018), as well as with more dysfunctional personality traits (Wolz et al., 2016) and emotional regulation deficits (Carlson et al., 2018).

The literature analysing FA and response to treatment is very scarce, and results are not yet conclusive. The few studies assessing the relationship between FA and treatment outcome in ED have focused on brief interventions for patients with BN (Hilker et al., 2016) or on a food-specific inhibition training for patients with BED (Giel, Speer, Schag, Leehr, \& Zipfel, 2017). In this vein, Hilker et al. (2016) found that severity of FA was a short-term predictor of abstinence from binging/purging episodes after a brief psychoeducational therapy among patients with BN (Hilker et al., 2016). However, other study applying food-specific inhibition training in patients with BED found that this intervention improved the inhibitory control towards highcaloric food stimuli, but it was not able to produce changes in food craving or FA in these patients (Giel et al., 2017). Additionally, the findings regarding FA and weight loss interventions in patients with obesity are still controversial. Although some authors found that FA may act as a predictor of less weight loss after a low-calorie dietetic intervention (Guerrero Pérez et al., 2018), others have shown that the presence of FA before surgery was not associated with presurgical body mass index (BMI) neither postoperative weight loss (Ivezaj, Wiedemann, \& Grilo, 2017). Furthermore, some studies have revealed that the weight loss induced by a low-calorie diet (Guzzardi et al., 2018) or by bariatric surgery (Murray, Tweardy, Geliebter, \& Avena, 2019; Pepino, Stein, Eagon and Klein, 2014; Sevinçer, Konuk, Bozkurt and Coşkun, 2016) induced remission of FA 
symptoms and improved several dietary behaviours associated with FA.

Thus, on the basis of the available evidence abovementioned, we hypothesized that FA may act as an indirect measure of ED psychopathology and, therefore, it may be associated with poorer treatment outcome in patients with binge disorder, namely BN or BED. Therefore, and taking into account all the aforementioned gaps in the literature, the specific aims of the study were (a) to analyse whether the presence of FA is associated with greater ED psychopathology in the different binge diagnoses (BN and BED) and (b) to analyse whether the presence of FA is associated with poor treatment outcome in patients with binge disorder, namely $\mathrm{BN}$ and $\mathrm{BED}$, after an outpatient cognitive-behavioural therapy (CBT) intervention.

\section{2 | METHODS}

\section{1 | Participants and procedure}

Sample composed of 71 adult patients diagnosed with binge disorders (42 BN and $29 \mathrm{BED}$ ), who were consecutive referrals to the Eating Disorders Unit at Bellvitge University Hospital in Barcelona. ED diagnoses were made by means of a face-to-face semistructured clinical interview (Fernández-Aranda \& Turón, 1998) that has been updated based on the validated SCID-5 interview (First, Williams, Karg, \& Spitzer, 2015) and conducted by experienced clinical psychologists and psychiatrists. The inclusion criteria for the sample were as follows: (a) 18 years and older, (b) diagnosed with BN or BED according to DSM-5 criteria; (DSM-5; APA, 2013), and (c) have completed a structured outpatient CBT group. Patients who dropped out the treatment were excluded from the present study (no statistical differences were found in the comparison between patients who completed and dropped out the CBT intervention, nor for the presence of a FA positive screening score $[P=.100]$, nor for the FA severity level [ $p=.443]$, nor for the ED severity level $[p=.519]$, nor for the age of onset of the $\operatorname{ED}[p=.279]$, nor for the duration of the disorder $[P=.235]$, and neither for the disorder subtype $[p=.766])$.

The present study was approved by the Ethics Committee of our institution (the Ethics Committee of Clinical Research of the Bellvitge University Hospital), and all research was conducted in accordance with the latest version of the Helsinki Declaration. Signed informed consent was provided by all participants.

\section{2 | Assessment}

Information about current ED symptoms, antecedents, and other psychopathological data of interest, as well as other relevant sociodemographic and clinical variables, were obtained by a face-to-face, standardized, structured interview. Additionally, the following commonly applied questionnaires in the field of EDs were employed:

- Eating Disorders Inventory-2 (EDI-2; Garner, 1991). The Spanish validation of this questionnaire has been used (Garner, 1998) to assess psychological and behavioural characteristics of the ED. The internal consistency (coefficient alpha) for the current sample was excellent $(\alpha=.94)$.

- YFAS (Gearhardt et al., 2009; Spanish validation (Granero et al., 2014). This is a 25-item self-report instrument that was designed to assess FA according to the seven symptoms of substance dependence listed in the Diagnostic and Statistical Manual of Mental Disorders 4th edition Text Revision. When at least three criteria and significant clinical impairment are reported, a diagnosis of FA is given. Moreover, a symptom count, indicating FA severity, can be measured to provide a score between 1 and 7 . The internal consistency of our sample was good $(\alpha=.84)$.

\section{3 | Treatment}

Treatment for BN and BED consisted of 16 weekly outpatient group therapy conducted by experienced psychologist. Patients with $\mathrm{BN}$ and $\mathrm{BED}$ were placed in separated therapy groups, but both treatment groups were based on the same CBT programme. The treatment protocol was manualized and published in Spanish (Fernández-Aranda \& Turón, 1998). Its effectiveness has been described previously (Agüera et al., 2013).

Patients were reassessed at discharge and categorized into the following three categories: full remission, partial remission, and nonremission. These categories were based on the consensus judgment of the senior clinical staff who considered all aspects of the patient's treatment outcome, such as frequency of binge episodes and compensatory behaviours (such as self-induced vomiting or laxative and diuretics misuse), and improvement in ED cognitions. To assess clinical significance of response to treatment, the therapists had access to objective primary measures, such as the number of binging/purging episodes per week, based on entries from a food diary. According to DSM-5 criteria (APA, 2013), the working definition of full remission was a total absence of 
symptoms meeting diagnostic criteria for at least four consecutive weeks, partial remission was defined as substantial symptomatic improvement but with residual symptoms, and the patients who presented poor outcomes were defined as nonremission. These categories were previously used to assess treatment outcome in threshold ED in other published studies (Agüera et al., 2015; Agüera et al., 2017; Custal et al., 2014; Sauchelli et al., 2016; Steward et al., 2016).

\section{4 | Statistical analysis}

Statistical analysis was carried out with Stata16 for windows. Partial correlations adjusted by sex and age estimated the association between the FA severity level with the other eating clinical variables (EDI-2 scores, BMI and frequency of binge eating episodes). In this analysis and due the strong association between statistical significance for the R-coefficients and sample size (low-poor coefficients tend to achieve statistical significance estimated into large samples, whereas high coefficients tend to nonstatistical significant into samples with a few number of participants), effect size was considered low-poor for $|R|>.10$, moderate-medium for $|R|>.24$ and large-high for $\mid$ $R \mid>.37$ (these thresholds corresponds to Cohen's $d$ of $0.20,0.50$, and 0.80, respectively (Rosnow \& Rosenthal, 1996).

Logistic regression assessed the contribution of the participant's age, the diagnostic subtype (BED vs. BN), the ED severity (EDI-2 total score), and the presence of FA (present vs. absent) on the likelihood of a CBT poor treatment outcome (defined as nonremission). Fitting of the model was valued with the Hosmer-Lemeshow test (goodness-of-fit was considered for $p>.05$ ); global predictive capacity was measured with the Nagelkerke's pseudo- $R^{2}$ coefficient, and global discriminative ability with the area under the ROC curve (AUC; effect size for $\mathrm{AUC}<.65$ was interpreted as low-poor, for. $65<\mathrm{AUC}<.70$ moderate-medium, and for AUC $>.70$ large-high; Steyerberg et al., 2001).

Path analysis procedures modelled as a case of structural equation modelling (SEM) estimated the underlying mechanism (including mediational links, direct, and indirect effects) between the participants' age, the diagnostic subtype, the FA severity, and the ED severity on the likelihood of the CBT treatment outcome. In this work, the maximum-likelihood estimation method of parameter estimation was defined and fitting was tested through standard statistical measures: the chi-square test $\left(\chi^{2}\right)$, the root mean square error of approximation (RMSEA), Bentler's Comparative Fit Index (CFI), the Tucker-Lewis Index (TLI), and the standardized root mean square residual
(SRMR). Adequate goodness-of-fit was considered for (Barrett, 2007): $P>.05$ in the $\left(\chi^{2}\right)$, RMSEA $<.08$, TLI $>.90$, $\mathrm{CFI}>.90$, and $\mathrm{SRMR}<.10$. The global predictive capacity of the model was measured by the coefficient of determination (CD). The model was obtained for the women subsample $(n=61)$ because the low frequency of men in the sample did not allow fitting.

\section{3 | RESULTS}

\section{1 | Characteristics of the sample}

Most of the participants were women $(n=62,87.3 \%)$ and single $(n=41,57.5 \%)$. Participants in the BN group were younger (mean $=30.6$ years old, $S D=10.0$ ) compared with participants with BED (mean $=39.3$ years old, $S D=9.9 ; p=.001$ ), and the onset of the ED was also at an early age in the BN group (mean $=19.1$ years old $[S D=6.6]$ versus mean $=25.2$ years old $[S D=11.3]$, $p=.006)$. No differences between the groups emerged for the duration of the disorder (mean $=11.3$ years $[S D=10.7]$ versus mean $=15.3$ years $[S D=9.8]$, $p=.111$ ). Table $\mathrm{S} 1$ includes the description for all the measures of the study and the comparison between the ED subtypes (BN vs. BED).

\section{2 | Comparison of the FA measures between sexes}

All men in the study met clinical criteria, according to YFAS, for a positive screening score in the FA measure, and $n=54(87.1 \%)$ women also reported positive screening. However, the comparison of the difference was statistically nonsignificant ( $p=.128$, but this result must be interpreted with caution due the small sample size), but effect size was into the moderate-medium range $(|d|=0.54)$. Furthermore, the number of total DSM criteria for FA was higher for women (mean $=5.7, S D=1.3)$ compared with men (mean $=5.1, S D=1.2$; although the mean difference was statistically nonsignificant $[p=.182]$, the effect size was into the moderate-medium range $[|d|=0.51]$ ).

\subsection{Association of the FA severity with ED measures}

Table S2 includes the association between FA severity (number of FA criteria) and the EDI-2 scales (bulimia and impulse regulation scales and the total score), BMI, and frequency of binge eating episodes (partial correlations adjusted by sex and age). When considering the total sample, a positive correlation between the FA and the number of weekly binge eating episodes and the ED 
psychopathology (measured by EDI-2) was found. Into the BN group, FA level positively correlated with ED psychopathology (specifically with EDI-2 bulimia and total scores), BMI, and weekly frequency of binge eating episodes. For patients with BED, FA severity also positively correlated with the ED psychopathology and with the weekly frequency of binge eating episodes.

\section{4 | Baseline FA and therapy outcome among the ED subtypes}

Table 1 displayed the percentage of patients with or without FA at baseline according to each therapy outcome category (full remission, partial remission, and nonremission) among the ED subtypes (Figure 1 includes the bar chart with the linear showing linear/quadratic

TABLE 1 Association between FA at baseline and treatment outcome among ED subtypes

\begin{tabular}{|c|c|c|c|c|c|c|c|c|c|c|c|c|}
\hline \multirow[b]{2}{*}{$\begin{array}{l}\text { Food } \\
\text { addiction } \rightarrow\end{array}$} & \multicolumn{4}{|c|}{ Total sample } & \multicolumn{4}{|l|}{$\mathbf{B N}$} & \multicolumn{4}{|l|}{ BED } \\
\hline & $\begin{array}{l}\text { No } \\
n=8\end{array}$ & $\begin{array}{l}\text { Yes } \\
n=63\end{array}$ & $p$ & $|d|$ & $\begin{array}{l}\text { No } \\
n=5\end{array}$ & $\begin{array}{l}\text { Yes } \\
n=37\end{array}$ & $p$ & $|d|$ & $\begin{array}{l}\text { No } \\
n=3\end{array}$ & $\begin{array}{l}\text { Yes } \\
n=26\end{array}$ & $p$ & $|d|$ \\
\hline Full remission & $62.5 \%$ & $57.1 \%$ & .882 & 0.11 & $40.0 \%$ & $40.5 \%$ & .826 & 0.01 & $100 \%$ & $80.8 \%$ & .444 & $0.69 *$ \\
\hline Partial remiss. & $25.0 \%$ & $33.3 \%$ & & 0.18 & $40.0 \%$ & $48.6 \%$ & & 0.17 & $0 \%$ & $11.5 \%$ & & $0.51^{*}$ \\
\hline Nonremission & $12.5 \%$ & $9.5 \%$ & & 0.10 & $20.0 \%$ & $10.8 \%$ & & 0.26 & $0 \%$ & $7.7 \%$ & & 0.41 \\
\hline
\end{tabular}

Note. Bold: effect size into the moderate-medium $(|d|>0.50)$ to large-high range $(|d|>0.80)$.

Abbreviations: BED, binge eating disorder; BN, bulimia nervosa; BED, binge eating disorder; $|d|$ : Cohen's $d$ coefficient (absolute value) ED, eating disorder; FA, food addiction.

*Significant comparison (.05 level).

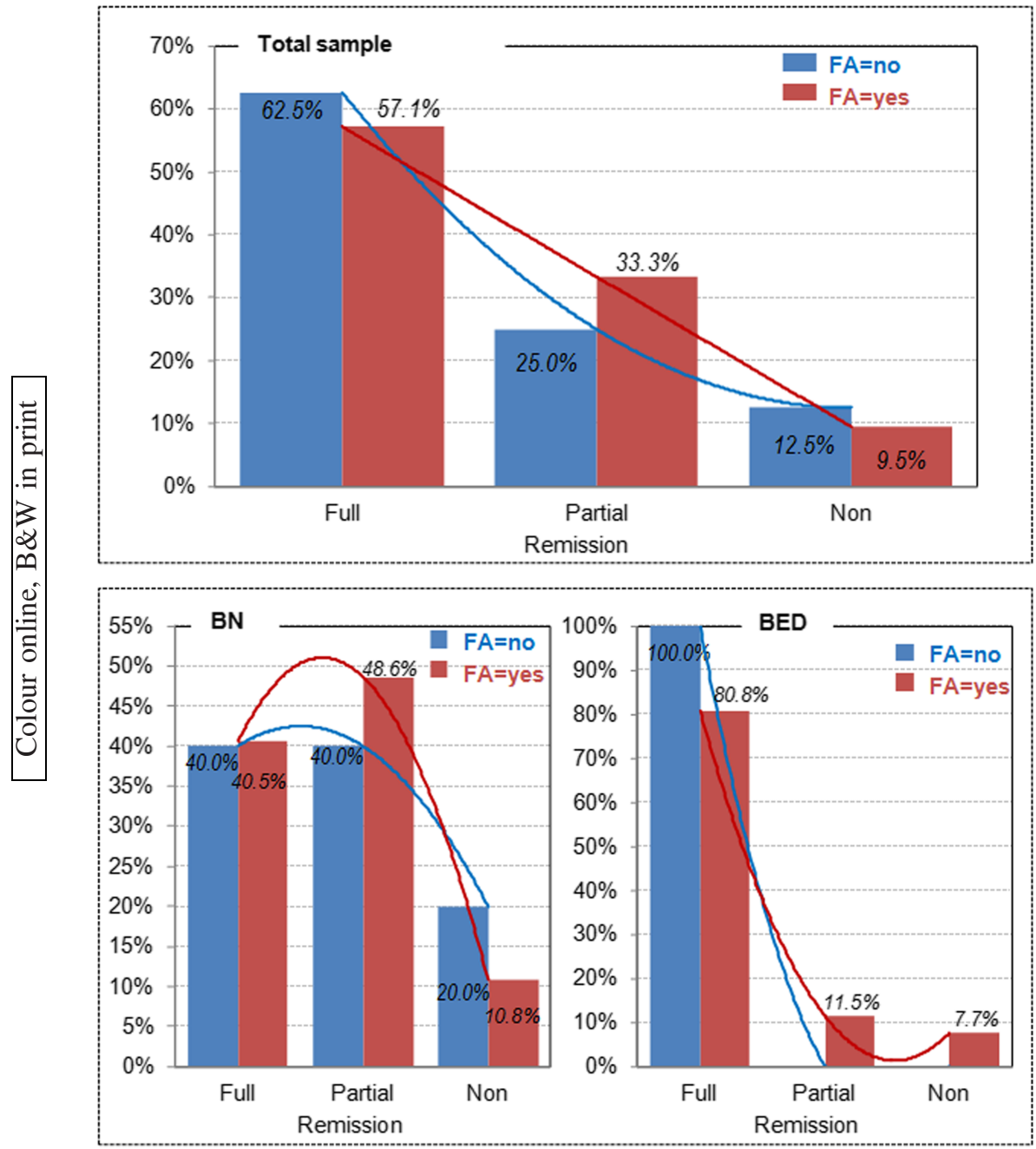

FIGURE 1 Bar-chart for the percentage of participants in each therapy outcome condition (full remission, partial remission, and nonremission; $n=71$ ). Note. FA, food addiction; BN, bulimia nervosa; BED, binge eating disorder. Blue colour line, linear/quadratic trend for the $\mathrm{FA}=$ no condition. Red colour line, linear/quadratic trend for $\mathrm{FA}=$ yes condition [Colour figure can be viewed at wileyonlinelibrary.com] 
trends). Whereas no differences between the groups were obtained in the resulting therapy outcome categories when considering the total sample and the BN subsample, there were significant differences obtained in the BED group. Namely, the participants with BED with baseline positive FA presented poorer treatment outcome than those with baseline negative FA ( $80.8 \%$ vs. $100 \%$ full remission, respectively).

\section{5 | Predictive model of the therapy outcome}

Table 2 contains the final logistic regression, when considered the whole sample (BN and BED), for the criterion therapy outcome "nonremission," considering as predictors the participants' age, diagnostic type, ED psychopathology (measured by means of EDI-2 total score), and presence of FA (positive vs. negative screening score). No significant interaction was found between the FA and the age $(p=0.347)$, suggesting that the effect of the FA on the therapy outcome was equal independent of the participants' age. The results of the model including the interaction parameter show that the risk of a poor therapy outcome is increased for older patients and those with a higher ED psychopathology at baseline. No predictive capacity on the therapy result was obtained for the diagnostic subtype and the FA. The model achieved good fitting (Hosmer-Lemeshow: $\boldsymbol{p}=.715$ ), large-high predictive capacity $\left(R^{2}=.225\right)$ and large-high discriminative ability (AUC $=0.833)$.

\section{6 | Pathways analysis (SEM)}

Figure 2 contains the path diagram with the standardized coefficients obtained in the SEM (complete results are in Table S3). The model only retained the significant coefficients, and adequate goodness-of-fit was obtained: $\chi^{2}=1.27(p=.259)$, RMSEA $=0.067$, CFI $=0.993$, $\mathrm{TLI}=0.935$, and SRMR $=0.031$. The results of the model showed that BED diagnosis and lower ED severity achieved direct effect on the CBT outcome, increasing the likelihood of full remission. Two mediational links also emerged: (a) older age was related to both lower ED severity levels and presence of BED diagnosis, which increased the risk of good outcome, and (b) lower FA severity were related to lower levels in the ED severity, which increased the odds of full remission. The global predictive capacity of the SEM was CD $=0.366$.

\section{4 | DISCUSSION}

Although the interest in studying the association between FA and ED has increased drastically in the last few years, most of the published studies have used cross-sectional

TABLE 2 Predictive capacity of FA level on the poor outcome (nonremission): Logistic regression $(n=71)$

\begin{tabular}{|c|c|c|c|c|c|c|c|c|c|}
\hline \multirow[b]{2}{*}{ Age (years) } & \multicolumn{6}{|c|}{ Coefficients } & \multicolumn{3}{|c|}{ Fitting } \\
\hline & $\begin{array}{l}\text { B } \\
0.106\end{array}$ & $\begin{array}{l}S E \\
0.049\end{array}$ & $\begin{array}{l}\boldsymbol{p} \\
.013^{*}\end{array}$ & $\begin{array}{l}\text { OR } \\
1.11\end{array}$ & 1.01 & 1.22 & $\begin{array}{l}\boldsymbol{R}^{2} \\
.225\end{array}$ & $\begin{array}{l}\text { AUC } \\
.833\end{array}$ & $\begin{array}{l}\text { H-L } \\
.715\end{array}$ \\
\hline Diagnosis $(0=\mathrm{BN} ; 1=\mathrm{BED})$ & -1.651 & 1.007 & .073 & 0.19 & 0.03 & 1.38 & & & \\
\hline ED severity (EDI-2 total) & 0.022 & 0.013 & $.045^{*}$ & 1.02 & 1.01 & 1.05 & & & \\
\hline
\end{tabular}

Abbreviations: AUC, area under ROC curve; BED, binge eating disorder; BN, bulimia nervosa; ED, eating disorder; FA, food addiction; H-L, Hosmer-Lemeshow test ( $p$ value); $R^{2}$, Nagelkerke's pseudo $R^{2}$.

*Significant parameter (.05 level).

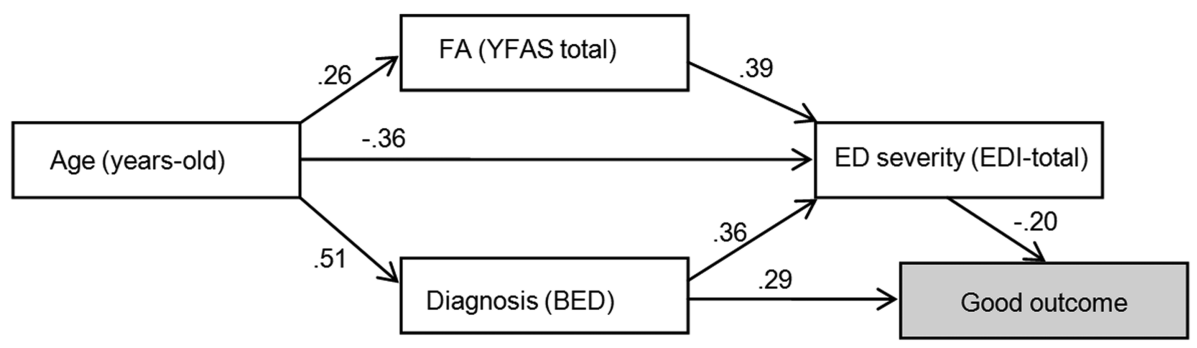

FIGURE 2 Structural equation modeling (SEM): path analysis obtained for the dependent variable "full remission" 
designs (Fernandez-Aranda, Karwautz, \& Treasure, 2018). One of the few studies analysing FA as a possible predictor of treatment outcome in patients with bulimic symptoms was carried out after a brief psychoeducational intervention (Hilker et al., 2016), and only the short-term effect was assessed. Hence, to our knowledge, this is the first study that attempted to address whether the presence of FA is associated with greater ED psychopathology and treatment outcome after a completed CBT outpatient programme.

First, as expected, this study confirmed previous findings on the high prevalence of FA in patients with BN (de Vries \& Meule, 2016; Meule et al., 2014) and BED (Gearhardt et al., 2014; Smith \& Robbins, 2013; Penzenstadler et al., 2018). Similar to previous studies (Granero et al., 2014), we found that about $87 \%$ of female patients with bulimic symptoms scored positive in the YFAS (Granero et al., 2014; Wiss \& Brewerton, 2017). Males with ED even had higher rates of FA than their female counterparts. These results are in disagreement with another study that indicated a higher prevalence of FA in women than in men (Hauck, Weiß, Schulte, Meule, \& Ellrott, 2017). This inconsistency might be explained by the fact that our study assessed patients with ED, whereas the study of Hauck et al. (2017) analysed FA in the general population. Therefore, our findings may be slightly inflated because of increased severity of eating-related symptoms in males with EDs. However, these results should be interpreted with caution due the small sample size of the males with ED's.

Second, the main finding of our study confirms the association between FA and the ED psychopathology. Patients with FA, both $\mathrm{BN}$ and BED, scored higher in the EDI-2 questionnaire and presented more weekly frequency of binge eating behaviours. These results are in line with previous literature that reported a direct association between FA, bingeing ED subtype and with greater eating and psychopathological severity (Granero et al., 2014). Some authors have even suggested that FA may be solely a marker of severity especially associated with factors such as more frequent binge eating episodes (Burrows et al., 2017; Burrows et al., 2018; Imperatori et al., 2014) and that FA does not independently measure addictive mechanisms (Granero et al., 2014; Hilker et al., 2016; Ivezaj, Wiedemann, Lydecker, \& Grilo, 2018). In addition, our results were not able to find significant differences between the presence of FA and BMI in ED, solely in those with BN. Hence, as suggested in previous studies (Villarejo et al., 2014), BMI may be a consequence of disinhibited eating behaviour rather than a factor related to FA itself. In ED patients, FA may not predict disordered eating behaviour indiscriminately, but it might be specifically linked to disinhibited eating (Burgess, Turan, Lokken, Morse, \& Boggiano, 2014).

Third, and partially supporting our hypothesis, our findings showed a direct association between the presence of FA and a poor clinical outcome in BED but not in BN or in the sample of ED as a whole. We found that the presence of FA in the subsample of patients with BED was associated with a lower likelihood of achieving full remission. Overall, patients with BED tend to show a better prognosis than patients with BN (Agüera et al., 2013; Linardon, Kothe, \& FullerTyszkiewicz, 2019). However, this finding suggests that the presence of FA in patients with BED might be acting as a maintenance factor for binge eating episodes, possibly due to its effect on the impaired reward system characteristic of addictive processes. The extent to which patients with BED can benefit from approaches and interventions based on an addictive model should be elucidated in further studies.

In terms of clinical predictors, our results showed an association between ED psychopathology and CBT outcome: The higher the ED psychopathology, the lower the probability of ED symptomatological remission. These results are in accordance with previous literature (Dakanalis, Colmegna, Riva, \& Clerici, 2017; Lammers, Vroling, Ouwens, Engels, \& van Strien, 2015; Vroling, Wiersma, Lammers, \& Noorthoorn, 2016; Wagner et al., 2015) reporting that ED psychopathology, such as binge eating episodes and shape/weight concerns, are significant predictors of dropout, and high levels of body dissatisfaction are associated with poor outcomes. In addition, our results also showed that age played an important role in the prognosis of these disorders. In line with previous studies, our results evidenced that younger patients presented with higher ED psychopathology (Agüera et al., 2017). Contrastingly, our findings indicated an association between older age and higher YFAS scores. These results are not in line with a previous research reporting that the younger population exhibited a higher prevalence of FA (Hauck et al., 2017). However, these discrepancies may be due to the fact that the study of Hauck et al. (2017) analysed people in the general population, whereas we assessed patients with binge disorders. Therefore, in our sample, older patients with binge disorders may be those with a longer duration of the disorder and increased FA symptomology (Hauck et al., 2017).

Finally, one of the most striking findings of our study was that FA per se was not directly associated with the response to treatment. These findings are not in accordance with a prior study reporting that FA severity is a short-term predictor of abstinence from binging/purging 
episodes in patients with $\mathrm{BN}$ after a brief group treatment (Hilker et al., 2016). However, although we were not able to find a direct effect of FA on the treatment outcome, FA seems to act as a mediator in the relationship between ED psychopathology (measured by the total EDI-2 score) and treatment outcome. In this sense, FA may act as a marker of the severity of the ED and, therefore, is the severity of the disorder (increased and impaired by the FA), rather than the presence of FA for itself, which acts as a predictor of nonremission.

\section{1 | Limitations and strengths}

Some limitations must be taken into account when interpreting the results of this study. First, we used the first version of YFAS (Gearhardt et al., 2009) that was developed to operationalize indicators of addictive-like eating, originally based on the Diagnostic and Statistical Manual of Mental Disorders 4th edition Text Revision criteria for substance-use disorders. However, it would be useful to replicate the results using the updated version of this scale (i.e., YFAS 2.0). The YFAS 2.0 was developed to reflect changes to diagnostic criteria in the DSM-5 (Gearhardt, Corbin, \& Brownell, 2016). Second, the limited sample size and especially the lack of males in the sample do not allow us to generalize the results. Third, the present study did not use the same standardized questionnaires at the end of the treatment (as objective measures), which would have contributed to the reliability and objectivity of our longitudinal findings. Finally, we were not able to analyse the presence of FA before the disorder, so we cannot confirm that FA came prior to the ED and, therefore, acts as a risk factor for the severity of the ED or, on the contrary, a greater severity of the disease causes greater FA presence, as a marker of severity.

Notwithstanding these limitations, the current study also has several strengths that should be noted. As far as we know, this is the first study assessing whether FA may act as a possible predictor of treatment outcome in patients with BN and BED. Also, the findings derived from this study might improve our ability to better understand the influence of FA in the prognosis of these patients and thereby aid in tailoring the best treatment alternatives.

\section{5 | CLINICAL IMPLICATIONS}

In the light of these findings, FA might be interpreted as a severity index of the ED indirectly associated with treatment outcome. Therefore, it would be necessary to determine which therapeutic tools might be effective for the treatment of FA, especially in patients with BED. In this sense, further studies are needed to assess the extent to which patients with BED can benefit from other approaches and interventions based on the addictive model to improve treatment outcomes (Meule, 2019). However, it is a controversial point because, despite the neurobiological and clinical similarities between FA and addictive disorders, the consumption of food (unlike alcohol, substances, or gambling) is necessary for life, making it impossible to design a treatment based on total abstinence.

\section{6 | CONCLUSIONS}

In conclusion, our findings confirm the high presence of FA in a clinical sample of EDs, concretely in bingeing ED subtypes. Our results also indicate that high FA levels were associated with greater ED psychopathology in patients with BED and BN. The presence of FA was found as a predictor of treatment outcome just in the case of BED but not in BN. Finally, the results suggest that FA may act as a marker of severity and, therefore, also as a mediator between ED severity and treatment outcome, especially for patients with BED.

\section{CONFLICT OF INTEREST}

The authors declare no conflict of interest.

\section{FUNDING INFORMATION}

We thank CERCA Programme/Generalitat de Catalunya for institutional support. This research was supported by Instituto de Salud Carlos III (ISCIII; grant numbers: PI14/00290 and PI17/01167), by Ministerio de Economía y Competitividad (grant number: PSI2015-68701-R), by Delegación del Gobierno para el Plan Nacional sobre Drogas (2017I067), by Ministerio de Sanidad, Servicios Sociales e Igualdad (grant number: PR338/17), by PERIS (Generalitat de Catalunya, SLT006/17/00077), and cofunded by FEDER funds/European Regional Development Fund (ERDF), a way to build Europe. CIBERObn and CIBERSAM are both initiatives of ISCIII.

The funders had no role in the study design, data collection and analysis, decision to publish, or preparation of the manuscript.

\section{ORCID}

Xandra Romero (D) https://orcid.org/0000-0003-4276-9793

Zaida Agüera (D) https://orcid.org/0000-0003-4453-4939

Roser Granero (D) https://orcid.org/0000-0001-6308-3198

Isabel Sánchez, (D) https://orcid.org/0000-0001-5874-8204 
Nadine Riesco (D) https://orcid.org/0000-0002-6336-4907

Susana Jiménez-Murcia (D) https://orcid.org/0000-0002-3596-

8033

Gemma Casalé (D) https://orcid.org/0000-0003-0765-4675

Isabel Baenas (1) https://orcid.org/0000-0001-7415-0616

Jose M. Menchon (D) https://orcid.org/0000-0002-6231-6524

Ashley N. Gearhardt (D) https://orcid.org/0000-0003-3843-

5731

Carlos Dieguez, (ID https://orcid.org/0000-0002-0919-4337

Fernando Fernández-Aranda (D) https://orcid.org/0000-0002-

2968-9898

\section{REFERENCES}

Agras, W. S., Crow, S. J., Halmi, K. A., Mitchell, J. E., Wilson, G. T., \& Kraemer, H. C. (2000). Outcome predictors for the cognitive behavior treatment of bulimia nervosa: Data from a multisite study. The American Journal of Psychiatry, 157(8), 1302-1308. https://doi.org/10.1176/appi.ajp.157.8.1302

Agüera, Z., Riesco, N., Jiménez-Murcia, S., Islam, M. A., Granero, R., Vicente, E., ... Fernández-Aranda, F. (2013). Cognitive behaviour therapy response and dropout rate across purging and nonpurging bulimia nervosa and binge eating disorder: DSM-5 implications. BMC Psychiatry, 13, 285. https://doi.org/10.1186/ 1471-244X-13-285

Agüera, Z., Romero, X., Arcelus, J., Sánchez, I., Riesco, N., JiménezMurcia, S., ... Fernández-Aranda, F. (2015). Changes in body composition in anorexia nervosa: Predictors of recovery and treatment outcome. PLoS ONE, 10(11), e0143012. https://doi. org/10.1371/journal.pone.0143012

Agüera, Z., Sánchez, I., Granero, R., Riesco, N., Steward, T., MartínRomera, V., ... Fernández-Aranda, F. (2017). Short-term treatment outcomes and dropout risk in men and women with eating disorders. European Eating Disorders Review, 25(4), 293-301. https://doi.org/10.1002/erv.2519

Albayrak, Ö., Wölfle, S. M., \& Hebebrand, J. (2012). Does food addiction exist? A phenomenological discussion based on the psychiatric classification of substance-related disorders and addiction. Obesity Facts, 5(2), 165-179. https://doi.org/10.1159/ 000338310

APA (2013). Diagnostic and statistical manual of mental disorders: DSM-5. Washington, DC: American Psychiatric Association.

Barrett, P. (2007). Structural equation modelling: Adjudging model fit. Personality and Individual Differences, 42(5), 815-824. https://doi.org/10.1016/j.paid.2006.09.018

Baucom, D. H., \& Aiken, P. A. (1981). Effect of depressed mood in eating among obese and nonobese dieting and nondieting persons. Journal of Personality and Social Psychology, 41(3), 577-585.

Bonder, R., Davis, C., Kuk, J. L., \& Loxton, N. J. (2018). Compulsive "grazing" and addictive tendencies towards food. European Eating Disorders Review, 26(6), 569-573. https://doi.org/10.1002/ erv. 2642

Burgess, E. E., Turan, B., Lokken, K. L., Morse, A., \& Boggiano, M. M. (2014). Profiling motives behind hedonic eating. Preliminary validation of the palatable eating motives scale. Appetite, 72, 66-72. https://doi.org/10.1016/j.appet.2013.09.016

Burrows, T., Hides, L., Brown, R., Dayas, C. V., \& Kay-Lambkin, F. (2017). Differences in dietary preferences, personality and mental health in Australian adults with and without food addiction. Nutrients, 9(3). https://doi.org/10.3390/nu9030285

Burrows, T., Kay-Lambkin, F., Pursey, K., Skinner, J., \& Dayas, C. (2018). Food addiction and associations with mental health symptoms: A systematic review with meta-analysis. Journal of Human Nutrition and Dietetics: The Official Journal of the British Dietetic Association, 31(4), 544-572. https://doi.org/10.1111/ jhn. 12532

Carlson, L., Steward, T., Agüera, Z., Mestre-Bach, G., Magaña, P., Granero, R., ... Fernández-Aranda, F. (2018). Associations of food addiction and nonsuicidal self-injury among women with an eating disorder: A common strategy for regulating emotions? European Eating Disorders Review: The Journal of the Eating Disorders Association, 26(6), 629-637. https://doi.org/10.1002/ erv.2646

Carter, J. C., Davis, C. (2011). An addiction model of binge eating disorder. In: Addiction medicine, Springer New York, p. 633-647.

Chao, A., Grilo, C. M., White, M. A., \& Sinha, R. (2014). Food cravings, food intake, and weight status in a community-based sample. Eating Behaviors, 15(3), 478-482. https://doi.org/ 10.1016/j.eatbeh.2014.06.003

Chao, A. M., Grilo, C. M., \& Sinha, R. (2016). Food cravings, binge eating, and eating disorder psychopathology: Exploring the moderating roles of gender and race. Eating Behaviors, 21, 41-47. https://doi.org/10.1016/j.eatbeh.2015.12.007

Custal, N., Arcelus, J., Agüera, Z., Bove, F. I., Wales, J., Granero, R., ... Fernandez-Aranda, F. (2014). Treatment outcome of patients with comorbid type 1 diabetes and eating disorders. BMC Psychiatry, 14, 140. https://doi.org/10.1186/1471-244X-14-140

Dakanalis, A., Colmegna, F., Riva, G., \& Clerici, M. (2017). Validity and utility of the DSM-5 severity specifier for binge-eating disorder. The International Journal of Eating Disorders, 50(8), 917-923. https://doi.org/10.1002/eat.22696

Davis, C. (2016). A commentary on the associations among 'food addiction', binge eating disorder, and obesity: Overlapping conditions with idiosyncratic clinical features. Appetite. https://doi. org/10.1016/j.appet.2016.11.001

de Vries, S.-K., \& Meule, A. (2016). Food addiction and bulimia nervosa: New data based on the yale food addiction scale 2.0. European Eating Disorders Review: The Journal of the Eating Disorders Association, 24(6), 518-522. https://doi.org/10.1002/ erv. 2470

Derogatis, L. R. (1992). SCL-90-R: Administration, scoring \& procedures manual-II for the (revised) version and other instruments of the psychopathology rating scale series. Clinical Psychometric Research, 1-16.

Fernandez-Aranda, F., Karwautz, A., \& Treasure, J. (2018). Food addiction: A transdiagnostic construct of increasing interest. European Eating Disorders Review: The Journal of the Eating Disorders Association, 26(6), 536-540. https://doi.org/10.1002/ erv. 2645 
Fernández-Aranda, F., \& Turón, V. (1998). Guia básica de tratamiento en anorexia y bulimia nerviosa. [handbook of anorexia and bulimia nervosa].

First, M. B., Williams, J. B. W., Karg, R. S., \& Spitzer, R. L. (2015). Structured clinical interview for DSM-5-Research version (SCID-5 for DSM-5, research version; SCID-5-RV) (pp. 1-94). Arlington, VA: American Psychiatric Association.

Fletcher, P. C., \& Kenny, P. J. (2018). Food addiction: A valid concept? Neuropsychopharmacology: Official Publication of the American College of Neuropsychopharmacology, 43(13), 2506-2513. https://doi.org/10.1038/s41386-018-0203-9

Garner, D. M. (1991). Eating disorder Inventory-2. Odessa: Psychological Assessment Resources.

Garner, D. M. (1998). Inventario de Trastornos de la Conducta Alimentaria (EDI-2)-Manual. Madrid: TEA.

Gearhardt, A. N., Boswell, R. G., \& White, M. A. (2014). The association of "food addiction" with disordered eating and body mass index. Eating Behaviors, 15(3), 427-433. https://doi.org/10.1016/ j.eatbeh.2014.05.001

Gearhardt, A. N., Corbin, W. R., \& Brownell, K. D. (2009). Preliminary validation of the yale food addiction scale. Appetite, 52(2), 430-436. https://doi.org/10.1016/j.appet.2008.12.003

Gearhardt, A. N., Corbin, W. R., \& Brownell, K. D. (2016). Development of the yale food addiction scale version 2.0. Psychology of Addictive Behaviors: Journal of the Society of Psychologists in Addictive Behaviors, 30(1), 113-121. https://doi.org/10.1037/ adb0000136

Gearhardt, A. N., White, M. A., Masheb, R. M., Morgan, P. T., Crosby, R. D., \& Grilo, C. M. (2012). An examination of the food addiction construct in obese patients with binge eating disorder. The International Journal of Eating Disorders, 45(5), 657-663. https://doi.org/10.1002/eat.20957

Gearhardt, A. N., Yokum, S., Orr, P. T., Stice, E., Corbin, W. R., \& Brownell, K. D. (2011). Neural correlates of food addiction. Archives of General Psychiatry, 68(8), 808-816. https://doi.org/ 10.1001/archgenpsychiatry.2011.32

Gendall, K. A., Sullivan, P. F., Joyce, P. R., \& Bulik, C. M. (1997). Food cravings in women with a history of anorexia nervosa. The International Journal of Eating Disorders, 22(4), 403-409.

Giel, K. E., Speer, E., Schag, K., Leehr, E. J., \& Zipfel, S. (2017). Effects of a food-specific inhibition training in individuals with binge eating disorder-findings from a randomized controlled proof-of-concept study. Eating and Weight Disorders: EWD, 22 (2), 345-351. https://doi.org/10.1007/s40519-017-0371-3

Gordon, E. L., Ariel-Donges, A. H., Bauman, V., \& Merlo, L. J. (2018). What is the evidence for "food addiction?" A systematic review. Nutrients, 10(4). https://doi.org/10.3390/nu10040477

Granero, R., Hilker, I., Agüera, Z., Jiménez-Murcia, S., Sauchelli, S., Islam, M. A., ... Fernández-Aranda, F. (2014). Food addiction in a Spanish sample of eating disorders: DSM-5 diagnostic subtype differentiation and validation data. European Eating Disorders Review: The Journal of the Eating Disorders Association, 22(6), 389-396. https://doi.org/10.1002/erv.2311

Granero, R., Jiménez-Murcia, S., Gearhardt, A. N., Agüera, Z., Aymamí, N., Gómez-Peña, M., ... Fernández-Aranda, F. (2018). Validation of the Spanish version of the yale food addiction scale 2.0 (YFAS 2.0) and clinical correlates in a sample of eating disorder, gambling disorder, and healthy control participants. Frontiers in Psychiatry, 9. https://doi.org/10.3389/ fpsyt.2018.00208

Guerrero Pérez, F., Sánchez-González, J., Sánchez, I., Jiménez-Murcia, S., Granero, R., Simó-Servat, A., ... Fernández-Aranda, F. (2018). Food addiction and preoperative weight loss achievement in patients seeking bariatric surgery. European Eating Disorders Review: The Journal of the Eating Disorders Association, 26(6), 645-656. https://doi.org/10.1002/erv.2649

Guzzardi, M. A., Garelli, S., Agostini, A., Filidei, E., Fanelli, F., Giorgetti, A., ... Pagotto, U. (2018). Food addiction distinguishes an overweight phenotype that can be reversed by low calorie diet. European Eating Disorders Review: The Journal of the Eating Disorders Association, 26(6), 657-670. https://doi.org/10.1002/ erv.2652

Hauck, C., Weiß, A., Schulte, E. M., Meule, A., \& Ellrott, T. (2017). Prevalence of 'food addiction' as measured with the yale food addiction scale 2.0 in a representative German sample and its association with sex, age and weight categories. Obesity Facts, 10(1), 12-24. https://doi.org/10.1159/000456013

Hilker, I., Sánchez, I., Steward, T., Jiménez-Murcia, S., Granero, R., Gearhardt, A. N., ... Fernández-Aranda, F. (2016). Food addiction in bulimia nervosa: Clinical correlates and association with response to a brief psychoeducational intervention. European Eating Disorders Review: The Journal of the Eating Disorders Association, 24(6), 482-488. https://doi.org/10.1002/ erv. 2473

Imperatori, C., Innamorati, M., Contardi, A., Continisio, M., Tamburello, S., Lamis, D. A., ... Fabbricatore, M. (2014). The association among food addiction, binge eating severity and psychopathology in obese and overweight patients attending lowenergy-diet therapy. Comprehensive Psychiatry, 55(6), 1358-1362. https://doi.org/10.1016/j.comppsych.2014.04.023

Ivezaj, V., Wiedemann, A. A., \& Grilo, C. M. (2017). Food addiction and bariatric surgery: A systematic review of the literature. Obesity Reviews : An Official Journal of the International Association for the Study of Obesity, 18(12), 1386-1397. https://doi.org/ 10.1111/obr.12600

Ivezaj, V., Wiedemann, A. A., Lydecker, J. A., \& Grilo, C. M. (2018). Food addiction among Spanish-speaking Latino/as residing in the United States. Eating Behaviors, 30, 61-65. https://doi.org/ 10.1016/j.eatbeh.2018.05.009

Jiménez-Murcia, S., Granero, R., Wolz, I., Baño, M., Mestre-Bach, G., Steward, T., ... Fernández-Aranda, F. (2017). Food addiction in gambling disorder: Frequency and clinical outcomes. Frontiers in Psychology, 8, 473. https://doi.org/10.3389/ fpsyg.2017.00473

Keser, A., Yüksel, A., Yeşiltepe-Mutlu, G., Bayhan, A., Özsu, E., \& Hatun, S. (2015). A new insight into food addiction in childhood obesity. The Turkish Journal of Pediatrics, 57(3), 219-224.

Lammers, M. W., Vroling, M. S., Ouwens, M. A., Engels, R. C. M. E., \& van Strien, T. (2015). Predictors of outcome for cognitive behaviour therapy in binge eating disorder. European Eating Disorders Review: The Journal of the Eating Disorders Association, 23(3), 219-228. https://doi.org/10.1002/erv.2356

Linardon, J., Kothe, E. J., \& Fuller-Tyszkiewicz, M. (2019). Efficacy of psychotherapy for bulimia nervosa and binge-eating disorder on self-esteem improvement: Meta-analysis. European Eating 
Disorders Review, 27(2), 109-123. https://doi.org/10.1002/ erv. 2662

Meule, A. (2019). A critical examination of the practical implications derived from the food addiction concept. Current Obesity Reports, 8(1), 11-17. https://doi.org/10.1007/s13679-019-0326-2

Meule, A., von Rezori, V., \& Blechert, J. (2014). Food addiction and bulimia nervosa. European Eating Disorders Review: The Journal of the Eating Disorders Association, 22(5), 331-337. https://doi. org/10.1002/erv.2306

Murphy, C. M., Stojek, M. K., \& MacKillop, J. (2014). Interrelationships among impulsive personality traits, food addiction, and body mass index. Appetite, 73, 45-50. https://doi.org/10.1016/j. appet.2013.10.008

Murray, S. M., Tweardy, S., Geliebter, A., \& Avena, N. M. (2019). A longitudinal preliminary study of addiction-like responses to food and alcohol consumption among individuals undergoing weight loss surgery. Obesity Surgery. https://doi.org/10.1007/ s11695-019-03915-3

Novelle, M. G., \& Diéguez, C. (2018). Unravelling the role and mechanism of adipokine and gastrointestinal signals in animal models in the nonhomeostatic control of energy homeostasis: Implications for binge eating disorder. European Eating Disorders Review, 26(6), 551-568. https://doi.org/10.1002/erv.2641

Obregón, A., Fuentes, J., \& Pettinelli, P. (2015). Association between food addiction and nutritional status in Chilean college students. Revista Médica de Chile, 143(5), 589-597. https://doi.org/ 10.4067/S0034-98872015000500006

Pedram, P., Wadden, D., Amini, P., Gulliver, W., Randell, E., Cahill, F., ... Sun, G. (2013). Food addiction: Its prevalence and significant association with obesity in the general population. PLoS ONE, 8(9). https://doi.org/10.1371/journal.pone.0074832

Pursey, K. M., Collins, C. E., Stanwell, P., \& Burrows, T. L. (2016). The stability of "food addiction" as assessed by the yale food addiction scale in a non-clinical population over 18-months. Appetite, 96, 533-538. https://doi.org/10.1016/j. appet.2015.10.015

Pursey, K. M., Stanwell, P., Gearhardt, A. N., Collins, C. E., \& Burrows, T. L. (2014). The prevalence of food addiction as assessed by the yale food addiction scale: A systematic review. Nutrients, 6(10), 4552-4590. https://doi.org/10.3390/nu6104552

Racine, S. E., Hagan, K. E., \& Schell, S. E. (2019). Is all nonhomeostatic eating the same? Examining the latent structure of nonhomeostatic eating processes in women and men. Psychological Assessment. https://doi.org/10.1037/pas0000749

Rosnow, R. L., \& Rosenthal, R. (1996). Computing contrasts, effect sizes and counternulls on other people's published data: General procedures for research consumers. Psychological Methods, 1, 331-340. https://doi.org/10.1037/1082-989X.1.4.331

Schulte, E. M., Avena, N. M., \& Gearhardt, A. N. (2015). Which foods may be addictive? The roles of processing, fat content, and glycemic load. PLoS ONE, 10(2), e0117959. https://doi.org/ 10.1371/journal.pone.0117959

Steward, T., Mestre-Bach, G., Agüera, Z., Granero, R., MartínRomera, V., Sánchez, I., ... Fernández-Aranda, F. (2016). Enduring changes in decision making in patients with full remission from anorexia nervosa. European Eating Disorders Review: The
Journal of the Eating Disorders Association, 24(6), 523-527. https://doi.org/10.1002/erv.2472

Steyerberg, E. W., Harrell, F. E., Borsboom, G. J. J. M., Eijkemans, M. J. C., Vergouwe, Y., \& Habbema, J. D. F. (2001). Internal validation of predictive models: Efficiency of some procedures for logistic regression analysis. Journal of Clinical Epidemiology, 54 (8), 774-781. https://doi.org/10.1016/S0895-4356(01)00341-9

Tomasi, D., Wang, G.-J., Wang, R., Caparelli, E. C., Logan, J., \& Volkow, N. D. (2015). Overlapping patterns of brain activation to food and cocaine cues in cocaine abusers: Association to striatal D2/D3 receptors. Human Brain Mapping, 36(1), 120-136. https://doi.org/10.1002/hbm.22617

Villarejo, C., Jiménez-Murcia, S., Álvarez-Moya, E., Granero, R., Penelo, E., Treasure, J., ... Fernández-Aranda, F. (2014). Loss of control over eating: A description of the eating disorder/obesity spectrum in women. European Eating Disorders Review: The Journal of the Eating Disorders Association, 22(1), 25-31. https://doi.org/10.1002/erv.2267

Volkow, N. D., Wang, G. J., Fowler, J. S., Tomasi, D., \& Baler, R. (2012). Food and drug reward: overlapping circuits in human obesity and addiction. Current Topics in Behavioral Neurosciences, 11, 1-24. https://doi.org/10.1007/7854_2011_169

Vroling, M. S., Wiersma, F. E., Lammers, M. W., \& Noorthoorn, E. O. (2016). Predicting dropout from intensive outpatient cognitive behavioural therapy for binge eating disorder using pretreatment characteristics: A naturalistic study. European Eating Disorders Review: The Journal of the Eating Disorders Association, 24(6), 494-502. https://doi.org/10.1002/erv.2474

Wagner, G., Penelo, E., Nobis, G., Mayrhofer, A., Wanner, C., Schau, J., ... Karwautz, A. (2015). Predictors for good therapeutic outcome and drop-out in technology assisted guided self-help in the treatment of bulimia nervosa and bulimia like phenotype. European Eating Disorders Review: The Journal of the Eating Disorders Association, 23(2), 163-169. https://doi.org/10.1002/ erv.2336

Weingarten, H. P., \& Elston, D. (1991). Food cravings in a college population. Appetite, 17(3), 167-175.

Westwater, M. L., Fletcher, P. C., \& Ziauddeen, H. (2016). Sugar addiction: The state of the science. European Journal of Nutrition. https://doi.org/10.1007/s00394-016-1229-6

Wiss, D. A., \& Brewerton, T. D. (2017). Incorporating food addiction into disordered eating: The disordered eating food addiction nutrition guide (DEFANG). Eating and Weight Disorders: EWD, 22(1), 49-59. https://doi.org/10.1007/s40519-016-0344y

Witt, A. A., \& Lowe, M. R. (2014). Hedonic hunger and binge eating among women with eating disorders. The International Journal of Eating Disorders, 47(3), 273-280. https://doi.org/10.1002/ eat.22171

Wolz, I., Hilker, I., Granero, R., Jiménez-Murcia, S., Gearhardt, A. N., Dieguez, C., ... Fernández-Aranda, F. (2016). "Food addiction" in patients with eating disorders is associated with negative urgency and difficulties to focus on long-term goals. Frontiers in Psychology, 7, 61. https://doi.org/10.3389/ fpsyg.2016.00061 


\section{SUPPORTING INFORMATION}

Additional supporting information may be found online in the Supporting Information section at the end of the article.
How to cite this article: Romero $\mathrm{X}$, Agüera $\mathrm{Z}$, Granero $\mathrm{R}$, et al. Is food addiction a predictor of treatment outcome among patients with eating disorder?. Eur Eat Disorders Rev. 2019;27:700-711. https://doi.org/10.1002/erv.2705 